\title{
Sarampión y Rubeola
}

\author{
POR EL DR. JULIAN ARCE \\ Profesor de Medicina Tropical en la Facultad de Medicina
}

En el año que acaba de terminar, se han observado en esta capital, además del recrudecimiento epidémico de la gripe, que no alcanzó felizmente las proporciones considerables que tuvo el año anterior (1918), una epidemia de sarampión y otra de rubeola, que se han desarrollado simultáneamente. Vamos a exponer, con tal motivo, algunas consideraciones sobre estas dos últimas enfermedades, cuya diferenciación clínica es, en muchos casos, muy difícil y no pocas veces imposible.

Pero, como al comenzar estas líneas hemos nombrado a la gripe, vale la pena que digamos, que continúan presentándose, con cierta frecuencia, casos no solo puros y por ende benignos de dichá infección, sino tambén complicados, septicémicos y violentos, de marcha fatál, como hemos tenido ocasión de ver hace pocos días (6-8 de febrero) en nuestra sala del hospital «Dos de Mayo", en un enfermo que presentó la cianosis heliutropo, descrita por algunos observadores en la influenza septicémica, con determinaciones broncopulmonares (1).

Diagnosticar el sarampión durante su desarrollo epidémico on lec niños no inmunes, no es, sin duda, difícil, pero no sucede lo mismo cuando reina, al mismo tiempo, en la localidad, la rubeola. afectando tanto a los que ya sufrieron antes el sarampión, como a los que no lo han sufrido todavía. Tal es el caso de Lima. Es cierto, que aún en esas condiciones, la identificación clínica del sarampión y de la rubeola, en los casos típicos, no presenta dificultades, pero,

(1) A. Agrahams, N. Hallows and H. French. A further investigation into influenzo-pneumococcal and influenzo-stroptococcal septicaemia.- The Lancets. 1919. Vol. 1. page. 1. 
como y! lado de dichos casos típicos, hay formas múltiples $j$ variadas, que aproximan anibas enlermedades hasta confundirlas on manifestaciones sintomáticas idénticas, el diagnóstico carece entonces de elementos diferenciales seguros $y$ su incertidumbre es mayer. todavía, si faltan clatos anamnésicos precisos respecto del saram. pión. De aquí, que los reglamentos sanitarios ingleses consideren desde 1915, no sólo el sarampión sino tantrién la ruteola, entre las enfermedades cuyá declaración es obligatoria.

Ultimamente, se han señalado dos medios auxiliares para distinguir dichas dolencias eruptivas, durante la evolución del exantema. Ae refiero a la prueiva de la ventosa y a la determinación de la fórmula leucocitaria. Én efecto, D OELSNitz. (1) desde 191 t se ha esforzado por encontrar un procedimiento de identificación precóz de los exantemas. en los casos dudosos, que fuese más preciso y más comparahle. que los procedimientos basados en la irritación del tegumento, dando la preferencia a uuna prueba mecánica*. Dospués de haber tentado, sin éxito, realizar esta prueba mecánica, por medio de la presión o el plegamiento prolongado de la piel, themos pensado, dice ese observador, en utilizar la acción aspirante de una ventosa sobre el tegumento sospechoso: la prueba o procedimiento de la ventosa nos ha parecido, desde entonces, reunir las ventajas que buscábamos". Empleada con resultados satisfactorios en el período pre-eruptivo del sarampión, lo ha sido, asímismo, con buen éxito, durante el período eruptivo, no sólo para el diagnóstico confirmatorio, sino también para el diferencial. "Así, dice D'OELsNirz, hemos podido distinguir ciertos eritemas toxi-alimenticios morbilifermes, en los cuales el resultado fué negativo; hemos observado erupciones de manchas rosadas lenticulares confluentes, que no reacionan a esta prueba; y sobre todo, hemos encontrado en el procedimient• de la ventosa un medio de distinguir el exantema de la rubeola de ciertas erupciones morbiliosas confluentes (algunas observaciones de M. GODLEWSKI vienen en apoyo de nuestras primeras comprobaciones a este respecto). Sobre la erupción de la rubeola, la ventosa no nos ha dado, habitualmente, ninguna acentuación regional del exantema. Este puede ser en nuestro concepto, un medio más de distinción entre esa fiebre eruptiva y el sarampión y la escarlatina, según los casos" En la erupción sarampionosa, la ventosa "refuerza la imagen eruptiva por una acentuación congestiva y aún equimotica del-exantema,persistiendo aquella largo tiempo bajo la forma de trazas pigmentadas que fijan más tarde el aspecto de la erupción ya

(1) M. D.Oelsnitz. Lépreuve de la veniouse dans leur sémiologique. La Presse Mt́dicale. 1919-pág. 268.

les fltores eruptives; sa ve- 
borrada, como una reliquia local que puede servir, útilmente, al diagnóstico retrospectivo de la afección".

Lesieur y JacQuet (1), estudiando las modificaciones de la formula sanguínea durante la evolución del sarampión y la rubeola, en el adulto, han llegado a comprobar ciertos hechos que, si se confirman, pueden tener gran valor práctico. En efecto, esos investigadores han examinado la sangre de 10 sujetos sarampionosos Y 15 rubeólicos; escalonados en un período epidémico de siete meses más - menos. Hemos tenido el cuidado, dicen, de escoger casos netos, de mediana intensidad y no complicados. de ambas enfermedades. Para caracterizar la ruteola, nos hemos basacio en la existencia de - denopatías numerosas y netas, en la ausencia o atenuación extrema del catarro oculo-nasal y, sol-re todo, en la ausencia de malestar premonitorio, habiendo caído enfermos los individuos al mismo tiempo que aparecía el exantema. Este último hecho, sobre todo, nos parece característico, porque no se vé casi, mayornente, sarampienes, por ligeros que seán, cuyo período de invasión trascurra enteramente inadrertido. La noción de epidemicidad no nos ha dado casi indicaciones. pues las dos enfermedades han estado en los casos presentes hátitualmente confundidas".

* Nos ha parecido, de una manera general, que la fórmula leucocitaria del sarampión, en el período de exantema, es uná fórmula banal de polinucleosis infecciosa; en la rubeola. por el contrario, la reacción es más bien del tipo linfoide, constituído por el predominio de los mononucleares, hecho que podía preveerse, desele luego, por la hiperplasia linfoide generalizada, bajo la forma de adenopatías múltiples, habitual en esta enfermedad".

Analizando esos autores las diferentes numeraciones leucocitarias que habían practicado, ohtienen las siguientes cifras: sarampión, 5 cusos de 9, con polinucleosis. 2 casos con equilibrio normal y 2 con mononucleosis lijera; rubeola, II casos de 15, con monunocleosis neta, 2 con equilibrio normal y 2 con polinucleosis. *El aumento de la cifra de los mononucleares, agregan, comprobado en la rubeola, corresponde, sobre todo, a las formas medianas. Los mononucleares medianos predominan, en efecto, en las láminas y constituyen casi los dos tercios de la cifra total de mononucleares.

"Entre estos elementos existen siempre en número apreciable, formas jóvenes de mononucleares, caracterizadas por su protoplasma escur•, netamente basófilo, que rodea el núcleo grueso, pálido y redondeado, según el tipo de los leucocitos incompletamente elabora-

1) Ch. Lesieur et PaUl JACQUeT.-De quelques jaits concernane la rubéle: réctions sanguine et ganglionnaire. La Presse Medicales 1919-pág. 292. 
dos. Estos elementos atestiguan, en nuestra opinión, la existericia di una hiperactividad leucogenética del tejido linfoide; se trataría. pues, en estos casos, de mononucleosis verdadera por hipergénesis ! no de una mononucleosis ficticia por caída momentánea de la tasa vic los polinucleares. Hemos encontrado, además, en una lámina, uń mielocito neutrófilo, pero no normoblastos, ni ningún otro elemonto de la serie mielógena".

"Si de otro lado se considera las fechas de la enfermedad en qui se han practicado esos exámenes, se constata que es hacia el 3er. dí después del principio del exantema, que la mononucleosis es má constante Y más acentuadi. Ahora bien, es precisamente al 3er dí. según nuestras observaciones, que las adenopatías rubeólicas estall en su máximum; las reacciones linfoides de la sangre y de los tejido: ganglionares, parecen pues paralelas".

"Otro hecho particular que revelan nuestros exámenes, es id abundancia anormal en la rubeola, de polinucleares basófilos con granulaciones metacromálicas (labrocitos de Blanchard).... Tres veces hemos encontrados estos elementos el quinto día de la enfermedad. Hay, pues, una matzellen-leucocitosis lijera y bastante partcular del principio de la convalescencia y que nos parecc digna ic retener la atención«.

"Buscando, sistemáticamente, las adenopalías en un gran número de enfermos, hemos hecho algunas comprobaciones: es así que la adenoparía occipital, que se ha dado como uno de los buenos si nos de la rubeola, nos ha parecido muy inconstante y ausente en la mitad de los casos, máso menos. Mucho más frecuentes son las adicnopatías inguinales y axilares, que, por su situación misma, son eir. dentemente menos características, Por el contrario, la adenoparía supra-epitrocleana nos ha parecido casi constante y constituye, para nosotros, un signo de glan valor. Se sabe, en efecto, cuan raramente perceptible es estc ginglio en el estado normal; se le sentía clard. mente, en un laclo o en el otro o en los dos a la vez, en casi todos nuestros eniemos y su búsqueda, a nuestro parecer, no debe ser jamás clescuidada cuando se sospechid la rubeola*.

"Parecc. pues, en resumen. y contrariamente a otras asercioncs. que se pucde oponer. en cierta medida, la fórmula hematológica del sarampión a la de la rubeola, al monos durante el exantema, carac. terizándose el primero por una leucocitosis polinuclear banal, la sc. gunda, al contrario, por una leucocitosis mononuclear, sobre to de las formas nedianas, en relación con el impulso ganglionar in. comitante, acompuñándose además, frecuentemente, con una mat. zellen-leucocitosis al principio de la convalecencia". 
- Estas distinciones son absolutas y podrían servir corrientemente al diagnóstico diferencial de las dos enfermedades? No lo creemos. per nuestra parte. Entre el sarampión y una rubeola morbiliforme. se recurrirá prácticamente para csclarecer el diagnóstico a las adenopatías-sobre todo occipital y supra-epicrocleana.-.-al polimortismo frecuente de la erupción rubcólicá a la auscacia o poca intensidad cel catarro óculo-nasal $\because$ solve rodo : la ausencia de males-

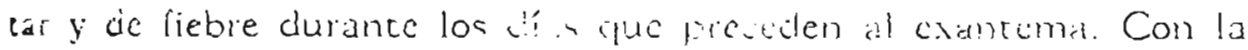
escurlatina, al contrario. el exámen de la simgre pudría prestar servicios, dada la polinucleosis, muy irancil y hicn conocida. de esta última enfermedad.

De los dos medios auxiliares a que se relicrun las interesantes contribuciones que acabamos cie trascribir. en sus partes más esenciales, la pruebáa de la rentosa parece tener. según D'OElsNitz valor piáctico definido. en el diagnóstico di!esencia! Jel sarampión y. rulseola, especialmonce cuando csl. última se presenta con fisonomía clínica ambigua. Lesieur ! J AcQueT. por su parte, creen en la utilidad del exámen de la sangre para diferenciar la rubeola de la escarlatina, pero no del sarampión, ! asignan gran impnitancia diagnostica a las adenopatías y a la ausencia de síntomas precursores del exantema para identificar la rubeola. Ahora bien, vamos a ver si en el terreno de la práctica, es positle, aún con los medios auxiliares apuntados, llegar a un diagnóstico firme. en los numerosos casos dudosos que nos ofrece la clínica. Para el efecto, clescribiremos tres casos que hemos tenido ocasión de observar en las últimas semanas, de los cuales el primero es un ejemplar cípico de rubcola. el segundo es un caso de mu ducloso carácter y el tercero. sin ser típico como el primero, es, sin embargo, facilmence diferenciable.

1. La señora : ..... de 52 años de cuad, blanca, casada, mulcíparia, sufrió durante su infanciá de sarampión intenso $y$, con excepción de varios ataques de angina ! de manilestaciones reumáticas su'r.oudas que en los últimos años se han repetido con liecuencia, ha gozado siempre de buena salud. diligiendo su hogar con celo y actividad constantes. Menopausia hacia los 48 años, con los trastornos inherentes a ese período crítico.

En los últimos días de dicicmlre próximo fasado, advirtió una mañana al despertar. cierto dolor en la nuca y en ambos lados del cue11. que se acentualia con los movimientos. No le dió importancia, atribuyéndolo a una probable mala posición de la cabeza en la almohada durante el sueño. Pero, al siguiente día (el 28 o 29), notó, con sorpresa, que el dolor era debido a que se habian inflamado y crecido los ganglios de la nuca y de las regiones laterales dol cuello, sin experimentar, por lo demás, ninguna otra molestia general o local, ni 
fiebre, ni disminución del apetito, $\mathrm{ni}$, en fin, desmedro de sus aiti. vidades habituales.

El 31, pude comprobar, en efecto, el aumento considerable de volumen de los ganglios occipitales y el infarto, en menor garde, te la cadena ganglionar que bordea el esterno-cleido-mastoideo, in sensibilidad tan esquisita en todos, que la enferma no permitía la palpación, aún lijera de dichos órganos. El 1. de enero, aparcí́ la explicación tácita del fenómeno, bajo la formà de un exantetha rubeólico en la cara, especialmente alrededor de la boca, en las meiillas y en la nariz. Los ojos estaban intensamente iny'ectados, tanto el globo como los párpados, acusando la enferma, como única molestia, sensación de sequedad de los ojos. Temperatura normal, buen apetito y, en general, todas las funciones en estado fisiológico. La enferma se resistía a considerarse tal y a confinarse en su dormitorio, ya que no aceptaba, de ningún modo, guardar cama.

La erupción se extendió lápidamente a los brazos y antebráos sobre todo, luego al pecho, vientre y por último a los miembros inferiores en más escasa proporción. Las mucosas, excepto la conjuntiva. permanecieron indemnes, no hahiendo en ningún momento, molestia, ni ardor en la garganta. Refiere la paciente, que había notado también, en los días anteriores al brote, junto con las primeras manifestaciones dolorosas localizadas en la nuca y el cuello, pesadí: ? abotagamiento de los párpados, con lijero dolor, presentándose después la inyección conjuntival con sensación mortificante de sequedad.

El exantema ofrecía en la cara aspecto escarlatinoso, por las manchas papulosas y eritematosas, de color rojo intenso, qui lo constituían y que resaltahan sobre el fondo blanco de la piel. En d tronco y los antebrazos sobre todo, la semejanza del rash con el de la escarlatina era igualmente notable, estando formado por elcm:ntos punteados, rojizos y lijeramente papulosos.

El 2 de enero, ocurrió a la enferma que al sentarse en el vasø de noche, experimentó un dolor tan vivo y desgarrador en las corius. que si no hubiese tenido al lado la tina del baño, en que se apo inmediatamente para levantarse, habría caído al suelo. El dolor, que se irradiaba a las pantorrillas, comprendía, asímismo, los hueses de las rodillas, con tal intensidad y carácter, que la paciente lo descrim diciendo, que sentía como si se le rompiesen las piernas. Dicho dolor. que recuerda el del dengue, fué decreciendo paulatinamente cn los días siguientes, a la vez que el brote, durando en total, más o menos. una semana.

Con la intensificacion del exantema en los antebrazos y manos. se produjo tumefacción y enrojecimiento considerables de las a tti. 
culaciones de los dedos, con dolor moderado y lijera incapacidad funcional, lo que no estorbó, sin embargo, el uso de las manos. Explorados los ganglios supra-epitrocleanos, se encontraron infartados, pero no dolorosos.

No obstante esos trastornos locales y la generalización del brote, la temperatura se mantuvo en los límites normales, sin ninguna repercusión sobre las demás funciones, que se conservaron intactas durante todo el curso del exantema.

El 7 de enero, la erupción se había marchitado, quedando, solamente, un punteado todavía apreciable en el tronco y antebrazos, que desapareció después de muchos días sin descamación aparente. La inyección conjuntival, la tumefacción de las manos y dedos, y los dolores de los miembros, desaparecieron, igualmente, hacia aquella fecha. El infarto ganglionar se resolvió con más lentitud, prolóngándose hasta fines de enero en que desapareció por completo.

Finalmente, atribuye la enferma el contagio a una vendedora ambulante de fruta, que tenía una erupción semejante a la que ella sufrio; pero hay que advertir, que en los últimos meses de 1919 grasó la rubeola, primero en las casas vecinas y después en los deparmentos contiguos de la misma casa ocupada por la paciente. La familia de ésta, consta de cuatro adultos y una criatura de trece meses de edad, ninguno de los cuales contrajo la infección.

Como se vé, este caso puede considerarse perfectamenre típico de la rubeola epidémica, inconfundible con el sarampión desde todo punto de vista, inclusive del exantema, que tuvo más bien cierta semejanza con el brote de la escarlatina, sin dificultar por ello el diagnóstico diferencial, que se impuso, puede decirse, desde los primeros momentos.

Pero como ya se ha dicho, no siempre sucede así y en muchas - cusiones, es sumamente difícil pronunciarse acerca de la naturaleza de la enfermedad, cuyos caractéres tanto pueden corresponder al sarampión como a la rubeola, dejando por consiguiente en el ánimo del médico la mayor intertidumbre.

2. $\mathrm{M}$. S., indio, natural de Chincha alta, de 19 años, jornalero, soltero, ingresó al hospital *Dos de Mayo* el 6 de enero del presente año, ocupando la cama N. 38 de nuestra sala. Refiere, que durante su infancia, sólo recuerda haber sufrido de diarreas y que nunca tuvo sarampión. A los doce años salí́ de su pueblo y se vino a esta capital, de donde pasó a la hacienda $\propto$ Infantas* a trabajar como peon. Después de año y medio de residencia en ese fundo, durante cuyo tiempo padeci6, una que otra vez, de catarro y tos, así como de epistaxis, en ocasiones abundante, se trasladó a la hacienda *Gallinazos". conde trabajo dos años, sufriendo también de catarros, tos y epis- 
taxis, como en «Infantas". Contrajo además un chancro con bubones supurados, cuya curación duró seis meses. Pasó enseguida al fundo «La Molina" y dos años después volvi6 a *Gallinazos", de donde ha venido con su actual enfermedad. En ambos lugares, siguib padeciendo de catarros y tos y además de epistaxis en «La Molina".

El 3 de enero, hasta cuya fecha había estado completamente bien, amaneció con malestar general, fiebre, dolor a la cabeza y los huesos, estornudos repetidos, ardor a la garganta, tos, eructos y sudores lijeros. No obstante esos síntomas, hizo su trabajo diario, intensificándose aquellos al día siguiente, especialmente la tos, y acompañándose de vómitos en la noche, que, asegura el enfermo, la pasó fuera de su lecho. El 5, con los síntomas anteriores, inclusive el vómito, y con diarreas copiosas de color amarillo, se vino a esta ciudad con el objeto de ingresar al hospital «Dos de Mayo», per como no lo consiguiese, se fué a pasar la noche a la hacienda "Chacra Cerro", de donde volvió al día siguiente en que fué récibido y alojado en la sala de "San Roque". Todos estos viajes los hizo el enfermo a pié. En la tarde del mismo día 5 , notó que había aparecido en la cara una erupción, que se generalizó rápidamente a todo el cuerpo. Su temperatura, en la tarde del 6 que ingresó al hospital, fué de $39 \circ 4 \mathrm{C}$

A la mañana siguiente que lo examinamos, estaba apirético (37\%) y: con el cuerpo cubierto de manchas eritematosas, más numerosas y marcadas en la cara. Los ojos se hallaban muy inyectados, casi sangrantes, tanto los globos como los párpados. La mucosa de las encías, mejillas, lengua y paladar, igualmente roja, así como la del relo del paladar, faringe y laringe, ocasionando disfagia y' voz y: tos roncas. En el dorso había algunas pústulas de acné y una erosión superficial en ambas piernas. Existía infarto ganglionar generalizado e indoloro, comprendiendo los ganglios supra-epitrocleanos. Hígado, aumentado de volumen, hasta tres traveses de dedo por debajo del reborde costal. Bazo, muy hipertrofiado y duro. Auscultando los pulmones, se percibía en ambos uno que otro estertor roncante. Corazón normal.

La erupción en este caso tenía aspecto morbilioso, sin ser típico En la boca el enrojecimiento no era en manchas o placas, sino difuso y muy intenso como ocurre en la rubeola.

El diario clínico consigna los siguientes datos: Día 9 -Uvula sumamente hiperemiada. Día 11-Ha desaparecido la gran inyeccción de las conjuntivas. Se nota muy apagado el brote de la cára Día 12-El enrojecimiento de la mucosa bucal y faringea, ha desaparecido. Persiste la ronquera. El exantema de la piel se percibe todavía, aunque muy apagado, habiéndose extinguido el de la cara. 
Bigeminación del pulso. Soplo sistólico en la punta del corazón. Día 13-Continúa la arritmia extrasistólica.

La temperatura se mantuvo normal, ascendiendo a $37^{\circ}$ en la tarde del 14 y a $37^{\circ} 2$ en la del 17. A su solicitud se le dí́ alta el 18, completamente curado del exantema. pero todavía ligeramente ronco.

El exámen de la sangre, practicado el 8 de enero, dió el el siguiente resultado:

Gl6bulos rojos: 4.440,000-Leucocitos: $;, 000$.

\section{Formula leucocitaria}

$\begin{array}{cccc}\text { Polinucleares neutrofilos } & - & 58 & \% \\ \quad \text { "' eosinófilos } & - & 4 & \text { ". } \\ \text { Formas de transicion } & - & 2 & \text { ", } \\ \text { Mononucleares grandes } & - & 0 & \text { ", } \\ \text { Linfocitos medianos } & - & 26 & \text { ", } \\ \text { Lino } & & - & 10\end{array}$

No hay formas anormales. ni se encuentra el hematozoario de Laveran.

Reacción de Wassermann, negativa.

Exámen bacteriológico del esputo: staphilococcus, streptococcus y micrococcus catarralis. Ausencia del hacilo de Koch.

El análisis de la orina sólo acusa de particular. trazas de serina y regular cantidad de inclicán.

La prueba de la rentosa practicada el 8 de enero, no dió ningún resultado sino después de cinco minutos de su aplicación, en que los elementos eruptivos se acentuaron. haciéndose casi equimóticos y permaneciendo en ese estado varios dias, de tal manera que eran todavía perfectamente visibles. cuando el resto del brote había desaparecido. Es decir, que esta prueba, según el concepto de. D’Oelnitz, favorecía el diagnóstico de sarampión.

3." El 9 de enero ingresó en nuestra sala. V. D., indio, natural de Arequipa, de 17 años, jornalero, soltero. ocupando la cama N.o 39 Interrogado acerca de sus antecedentes, manifiesta que ignora las enfermedades que haya podido tener en los primeros años de su vida, pues sus recuerdos más remotos sólo alcanzan a unas tercianas que sufrió a la edad de cuatro años: en la campiña de Arequipa de donde es oriundo, y que, con intervalos de tres o cuatro meses, se repitieron hasta que tuvo nueve años. Padeció también en esa misma época, de catarros frecuentes y prolongados; y a los ocho años tuvo una disentería que duró más de un mes. Vino después al Callao destinándose 
primero en una lechería donde sufrió de unos granos que le salian en la axila derecha, por cuyo motivo ingresó al hospital de "Guada lupe», de donde salió a los veinte días completamente curado, deatinándose entonces en una casa como doméstico. Allí permaneció año y medio, sufriendo como antes de catarros frecuentes; al cabo de ese tiempo se fué a trabajar a la hacienda "Oquendo" donde ha permanecido hasta el presente. En dicho fundo ha padecido continuamente de tercianas, las que desaparecían tres o cuatro meses, para reaperecer después con los síntomas consiguientes, asociados a epistaxis repetidas. Ha sufrido, además, en dos ocasiones, de cólions y también de calambres muy intensos.

El 7 de enero, amanecí́ con malestar general y fiebre lijera, que no le impiedieron salir a trabajar, notando entonces que tenía unas manchas en la cara. El 8, continuaron los síntomas anteriores más acentuados, acompañándose la erupción de la cara con inyección muy intensa de los ojos, molestia a la deglución y tos. Trabajo, sin embargo, ese día, creyendo, como decían varias personas, que tenía la gripe, pero como el 9 se sintiese peor $y$ las manchas se extendiesen a todo el cuerpo, emprendó viaje a esta capital, consiguiendo ingresar al hospital "Dos de Mayo". Su temperatura en la tarde de we día, fué de $36^{\circ} 7 \mathrm{C}$.

En la visita del 10, encontramos al enfermo en decúbito dorsal, llamando inmediatamente la atención, el enrojecimiento intenso de los ojos, que parecían estar a punto de verter sangre, y las manchas rojizas de la cara con descamación furfurácea abundante. Descubierto el cuerpo, se observó que la crupción cubría copiosamente la superficie del torax y miembros superiores, en menor proporción los miembros inferiores. Las manchas, con lijero relieve sobre la piel sin ser propiamente pápulas, tenían coloración más intensa en el centro y sus contornos no parecian muy definidos.

Las mucosas ocular y buco-faringea, estaban considerablemente inyectadas. Ya hemos señalado el enrojecimiento intenso de la conjuntiva óculo-palpebral. En idéntica condición se encontraba la mucosa oral, especialmente la de las encías, paladar, úvula, pilares y amigdalas, acompañándose de disfagia. El enantema visible en la faringe, se propagaba, sin duda, a la laringe y traquea, ocasionando ronquera y tos.

Los ganglios del cuello estaban infartados y dolorosos a la presión, así como los ganglios supra-epitrocleanos que tenían las dimensiones de un grano de trigo.

La auscultación de los pulmones demostró la existencia de un• que otro estertor roncante. Los demás órganos $y$ aparatos, no pre- 
sentaban alteraciones apreciables. El enfermo acusaba debilidad $y^{\prime}$ abatimiento marcados.

Practicada la prueba de la ventosa, se pudo apreciar, al cabo de un minuto, una acentuación intensísima del exantema, cuyas manchas tomaron aspecto hemorrágicn. tal como lo describe D' OELSNITz en el sarampión.

La marcha de la enfermedad, según el diario clínico. fué la siguiente: Día 11 -Continúa abundante e intensa la erupción en la piel. La mucosa de la hóveda palatina presenta un color rojo escarlata, lo mismo que los pilares y las amígdalas hipertrofiadas. Hay estertores subcrepitantes diseminados en ambos pulmones.

Exámen de la sangre:

Glóbulos rojos - 4.050,000. Leucocitos - 11.000.

Fórmula leucocitaria:

\begin{tabular}{|c|c|}
\hline Polinucleares neutrófilos & - \\
\hline eosinófilos & \\
\hline Formas cle transición & - \\
\hline Mononucleares grandes & \\
\hline medianos & - \\
\hline
\end{tabular}

Ausencia del hematozoario de Laveran.

Examen de la orina: nada de anormal.

Exámen del espulo: hacilo de Pfeiffer $\because$ micrococcus catarralis.

Día 12-Las conjuntilas están todavía muy congestionadas. El enrojecimiento del hrote va disminuyendo. Persisten las manchas equimóticas producidas por la ventosa.

Día 13-Disminuye la hiperemia de la mucosa bucal : continúan la ronquera y la tos.

Día 14--El brote cutáneo marchito. el enamtema apenas perceptible.

Día 15-Descamación fina en el cuerpo

La temperatura se mantuvo normal con excepción de los días $14,15,16$ y 19 de enero, que álcanzó en la tarde 3705, 37०, 37०2, y $37 \cdot 2$, respectivamente, El $1^{\circ}$ de febrero salió el enfermo del hospital completamente curado, habiencio prolongado su permanencia hasta esa fecha, por motivos independientes de la rubeola.

El diagnóstico en este caso no puede considerarse verdaderamente difícil, si se tiene en cuenta los siguientes hechos que hablan en favor de la rubeola: 1. 'la aparición del brote el mismo día en que se inició la enfermedad; $2 .^{\circ}$ el carácter benigno y la evolución api- 
rética del exantema, no obstante el compromiso de la mucosa de las vías digestivas y respiratorias superiores; es cierto que el enfermo refiere que tuvo fiehre lijera los dos días anteriores a su ingreso en el hosptal, pero dpalte de que esa afirmación cs un tanto vaga, ella no afecta el diagnóstico de rubeola, puesto que la pirexia se produjo el mismo día que apareció la erupción y no antes, como ocurre en el sarampión; $3 .^{\circ}$ la ausencia de catarro óculo-nasal: y $4 .^{\circ}$ el infarto doloroso de los ganglios cervicales y supra-epitrocleanos, adenopatía esta última, que, como se ha visto. tiene para LEsIEUR y JACQUET gran valor diagnóstico.

Pero, a pesar de esos hechos que podemos llamar afirmativos, hay algunos otros que pueden calificarse de dudosos y que hacen que este caso no sea tan evidente e inconfundible, como el primero de los que hemos descrito. En primer lugar, falta el antecedente de un ataque anterior de sarampión, aunque es muy probable que el enfermo haya sufrido esa dolencia en los primeros cuatro años de su vida de que no conserva ningún recuerdo, ni tiene ningún dato; es sabida, en efecto, la endemo-epidemicidad del sarampión y su frecuencia en nuestra población infantil, lo que inclina a aceptar la fundada probabilidad de que haya padecido el sujeto esa fiebre eruptiva durante su infancia. El compromiso del árbol respiratori (ronquera, tos, estertores), favorece igualmente la hipótesis del sarampión, pero estos trastornos leves, exentos de fiebre, y aún con esta, no son raros en la rubeola de los adultos, ya que en los niños pueden llegar esos disturbios hasta la bronconeumonia, según le afirman los autores.

Ahora bien, estahlecido el diagnóstico de rubeola, la prueba de la ventosa practicada en este caso, resulta infiel, si nos atenemos al criterio de D'OELSNItz, para quien la acentuación congestiva y aún equimótica del exantema. que persiste largo tiempo bajo la forma de trazas pigmentadas, no solo puede servir para afirmar su naturaleza sarampionosa, dacla lá ausencia de ese fenómeno en la rubeola, sino también para hacer el diagnóstico recrospectivo de la infección morbiliosa. En nuestro enfermo se obtuvo, en efecto, esa prueha positiva, con todos los caracteres indicados, en el brevísimo tiempo de un minuto, que háce todavía más notable el resultade, y con la persistencia prolongada de las manchas y sus trazas pigmentadas consecutivas

La fórmula leucocitaria tampoco ha correspondido a la que señalan LESIEUR y J ACQLeT á la rubeola en el período de la erupción, pues examinada la sangre al cuarto día del exantema, que es cuando las adenopatías rubeólicas están en su máximum y la mononucleosic según esos observadores, es más constante y acentuada, se compro- 
bó una leucocitosis polinuclear banal, que es la fórmula leucocitaria del sarampión.

El caso $2 .^{\circ}$, a diferencia de los casos $1 .^{\circ}$ y $3 .^{\circ}$, es, evidentemente, de diagnóstico difícil y por consiguiente discutible. Se trata, en efecto, de una pirexia eruptiva, que se inicia con malestar general, cefalalgia, dolores óseos, catarro nasal, disfagia, tos, vómitos, diarreas y que en la tarde del tercer día revela su exantema, primero en la cara y rápidamente después en todo el cuerpo. Declina entonces la temperatura hasta la normal y el brote, que había ganado también la mucosa buco-faringea pero en lorma difusa, se marchita gradualmente y desaparece al cábo de varius días. El exantema tuvo en la piel mucha semejanza con el del sarampión, no así en las mucosas donde presentó las caractéres del rubeólico. Ahora bien, si a estos datos se agrega la afirmación reiterada del enfermo de no haber sufrido jamás de sarampión y la existencia de esta enfermedad en forma epidémica, hay que reconocer que el platillo de la balanza se inclina hacia el lado de la pirexia morbiliosa.

La prueba de la ventosa fué asimismo favorable a ese diagnóstico, pero hubo de esperarse cinco minutos para que ella se produjese claramente, haciendo así contraste marcado en cuanto al tiempo y en cuanto a la intensidad, con lo que se observó en el caso $3 .^{\circ}$.

En cambio, la fórmula leucocitaria fué la de la rubeola, marcando una mononucleosis total de $38 \%$, en cuya cifra predominan los mononucleares medianos $(26 \%)$. El infarto de los ganglios cervicales y supra-epitrocleanos, habla también en favor de la rubeola, acentuando de este modo las dudas acerca de la verdadera naturaleza de la enfermedad.

En efecto, el exantema rubeólico, que, en la mayoría de los casos, aparece sin ser precedido de fiebre, lo es en otros, por uno, dos y aún tres días de pirexia, acompañada de los trastornos consiguientes, así como de catarro, disfagia y tos. En el curso de la última epidemia, se han observado no pocos casos de esa clase en adolescentes y adultos, que habían sufrido, años antes, de sarampión intenso y aún grave. La existencia de un período febril, pre-eruptivo, no puede considerarse, por consiguiente, como prueba absoluta conva el diagnóstico de rubeola. en el segundo de nuestros enfermos, aunque asegure este no haber padecido nunca de sarampión, negativa que por lo demás no tiene mucha fuerza, si se considera la ignorancia de esa clase de sujetos, que muchas veces no saben con pre- isión ni la edad que tienen, mucho menos las enfermedades que than sufrido durante los primeros años de su vida, entre las cuales se cuenta siempre o casi siempre, el sarampión que, como ya hemos dicho, es una de nuestras endemo-epidemias más comunes. 
Fundado en estas consideraciones he calificado este caso, com los anteriores, de rubeola, sin desconocer por ello la inseguridad del diagnóstico, muy discutible, sin duda, desde diversos puntos de vista.

Hace falta, pues, para zanjar esas dificultades diagnósticas, tan comunes en la práctica cuando reinan simultánea y epidémicamente el sarampión y la rubeola, precisar, hasta donde es posible, los síntomas y signos físicos más característicos de cada una de ellas, ya que la etiología de ambas permanece aún en el misterio. Desgraciadamente, no hay trastornos ni signos, inclusive el de Koplik, que puedan considerarse patognomónicos y que existan en todos los casos, sin excepción, o que perduren el tiempo suficiente para comprobar su existencia durante el curso de la enfermedad.

Quizá si el infarto ganglionar mejor estudiado, puede suministrarnos un medio seguro de diagnóstico, por más que exista tante en el sarampión como en la rubeola. En efecto, " las tonsilas, dice OSLER (1), describiendo el sarampión, y las glándulas linfáticas cervicales, pueden hincharse ligeramente y ser sensibles; algunas veces hay poliadenitis*. Refiriéndose a la rubeola, dice, igualmente: "Las glándulas linfáticas del cuello están hinchadas con frecuencia y cuando la erupción es muy intensa y difusa, el infarto se extiende a los ganglios de otras regiones del cuerpo".

Williams, avanza un poco más en la descripción de las alteraciones ganglionares, como se verá en seguida. Refiriéndose al sarampión dice: "Al segundo día la temperatura es más alta, la secreción de la nariz y naso-faringe es moco-purulenta y las glándulas linfáticas, especialmente las situadas detrás y debajo de la mandíbula inferior, están hipertrofiadas y sensibles». En la rubeola señala lo siguiente. "Es casi constante la hipertrofia de los ganglios, especialmente los cervicales posteriores, los situados detrás del ángul• de la mandíbula y los que corren a lo largo de los músculos esternomastoideos; en algunos casos, los ganglios de las axilas e ingles tanibién se encuentran hipertrofiados y sensibles, aunque por regla general el enfermo no acusa dolor. El aumento de volumen de los garglios del cuello es siempre un síntoma precóz y puede descubrírsele hasta una semana antes de la aparición del brote». (2)

Por último, LESIEUR y JACQUET, como se habrá visto, recalcan el valor diagnóstico de las adenopatías, especialmente la superepitrocleana, cuya constancia en la rubeola les parece sumamente importante.

(1) W. Osler. Practice of Medlcine, 1910-pags. 143 y 146

(2) Allbutt and Rolleston. System of Medicine, 1912-V. II. Part. I. pas: 389 y 106. 
Ahora bien, en los casos que hemos descrito, las adenopatías han sido constantes $y$ han interesado en todos, los ganglios supraepitrocleanos, hecho evidentemente significativo, dada la rareza clinica de este último infarto en las infecciones generales. Creemos, pues, con Lesiel'R y JACQLet, que ese fenómeno es digno de llamar la atención y que debe. por consiguiente, investigarse su existencia en todos los casos de ruteola. establecierido. hasta donde sea posible. sus relaciones con el exantema. Hace algunos años que investigamos sistemáticamente en nuestros enfermos no siflíticos, el estádo de los ganglios linfáticos, encontrando que es muy irecuente el infarto de los ganglios cervicales laterales e inguino-crurales; que es menos frecuente, pero no raro, el de los ganglios occipitales; y que es excepcional el de los supra-epitrocleanos. Solo hay una enfermedad en la que hemos encontrado, casi sin excepción, infarto general de los ganglios, inclusive los supra-epitrocleanos, y es la verruga peruana, en sus dos formas: benigna y maligna; con la circunstancia de que esa poliadenitis verrugosa, es una de las primeras manifestaciones de dicho proceso morboso.

El infarto de los ganglios del cuello, sobre todo de los occipitales, ha sido más estudiado, no sólo en cuanto a su intensidad, sido también en lo que se refiere al tiempo de su producción. La tumefacción de los granglios occipitales puede alcanzar, en efecto, al decir de algunos autores, proporciones considerables. Así, THOMson, asegura que la unica característica distintiva de la rubeola, es la tumefacción de las glándulas post-cervicáles, que pueden ser tan grandes como una nuez (walnut) (1). En nuestro primer caso, - tumefacción inflamatoria, dolorosa, de los ganglios occipitales, alcanzó casi las dimensiones de un garbanzo. Además en esa enferma, la adenitis cervical fué, como dice Williams, un síntoma precéz precediendo varios días al brote rubeólico. Sería, pues, muy util, establecer las relaciones, que sin duda existen en la rubeola, entre la aparición, intensidad y extensión de la adenitis y el exancoma correspondiente.

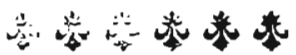

(1) W. H. Thomson. Clinical Medicine. 1919-pág. 96. 